\title{
Early post-stroke rehabilitation for upper limb motor function using virtual reality and exoskeleton: equally efficient in older patients
}

\author{
Tereza Gueye ${ }^{1,2,3}$, Miriama Dedkova ${ }^{2}$, Vladimir Rogalewicz ${ }^{3}$, \\ Marcela Grunerova-Lippertova ${ }^{2,4}$, Yvona Angerova ${ }^{3}$ \\ ${ }^{1}$ Stroke Rehabilitation Unit, Geriatrics Department, First Faculty of Medicine, Charles University in Prague, Czech Republic \\ ${ }^{2}$ Department of Rehabilitation Medicine, Faculty Hospital Kralovske Vinohrady, Prague, Czech Republic \\ ${ }^{3}$ Department of Rehabilitation Medicine, General University Hospital and First Faculty of Medicine, \\ Charles University in Prague, Czech Republic \\ ${ }^{4}$ Centre of Neurological Outpatient Rehabilitation ANR Bonn, Germany
}

\begin{abstract}
Aim of the study. To evaluate the effectiveness of virtual reality therapy (VRT) Armeo Spring ${ }^{\bullet}$ upper limb exoskeleton (Armeo), in early post-stroke rehabilitation with a focus on the elderly.

Clinical rationale for the study. Convalescence from a stroke is a complex process driven by a spontaneous recovery supported by multifactorial activation. Novel technology-based rehabilitation methods are being introduced to support brain plasticity.

Materials and methods. Using a randomised controlled study design, participants within 30 days after stroke with arm paresis were, in addition to a daily rehabilitation programme, assigned to an intervention group (45 minutes Armeo IG $n=25$; mean age 66.5 years) performing VRT, or to a conventional physiotherapy ( 45 minutes) control group (Armeo CG, $n=25$, mean age 68.1 years). Montreal Cognitive Assessment (MoCA), Functional Independence Measure (FIM) and Fugl Mayer Assessment Upper Extremity Scale (FMA-UE) were performed before and after the three-week therapy with 12 therapeutic sessions. Results of participants $<65$ and $\geq 65$ years old were compared.

Results. Paretic upper arm function improved significantly in both the IG and CG groups, the improvement in FMA-UE was significantly higher in the IG compared to the $C G(p=0.02)$, and patients $\geq 65$ years old presented an equal magnitude of improvement in paretic arm function compared to younger patients.

Conclusions and clinical implications. Early post-stroke rehabilitation strategies using, in addition to the daily rehabilitation programme, VRT with visual biofeedback is more effective on upper extremity motor performance than conventional physiotherapy, and the effectiveness does not diminish with patient age. This may be a promising addition to conventional physiotherapy in older stroke patients as well as in younger.
\end{abstract}

Key words: early neurorehabilitation, virtual reality, exoskeleton, upper limb, elderly, stroke (Neurol Neurochir Pol 2021; 55 (1): 91-96)

\section{Introduction}

Due to demographic ageing, the number of older stroke survivors is steadily increasing. Mortality and functional outcome depend primarily on rapid recognition of the stroke signs by the individual or his or her community, an organised system of care from ambulance to stroke unit, prompt evaluation by the stroke team, and the fast administration of reperfusion including intravenous thrombolysis (IVT) and endovascular thrombectomy (EVT) [1,2]. After this acute intervention, 
early rehabilitation based on neurorehabilitation principles contributes significantly to the optimisation of functional outcomes and overall well-being/quality of life of elderly stroke patients. Novel technology-based rehabilitation methods are being introduced to support brain plasticity. However, little is known about how these new technologies are accepted by older patients, or whether their effectiveness is comparable to that seen in younger individuals. Convalescence from a stroke is a complex process driven by a spontaneous recovery supported by multifactorial activation [3, 4]. From studies on neuroplasticity, we know that rehabilitation should start early, be intensive enough (in time or contain a high number of repetitions), and be focused on function, to achieve the best results [5-7].

These are the principles that therapies with different biofeedback, robot-assisted therapies (RT) or therapies using virtual reality (VR) are based upon. They motivate patients to do a higher number of repetitions, prolong the therapeutic time, and bring stimulation through their engaging design. In VR, virtual environments and objects provide the user with visual feedback, which can be presented through a head-mounted device, projection system, or flat screen. Biofeedback enables control on the timing of the task and supports motor learning [8].

\section{Clinical rationale for the study}

There have been numerous studies on the effectiveness of the use of RT or VRT by patients after stroke in subacute and chronic state, but only a few studies concerning the acute phase [9-11]. Acute phase studies usually explore RT as an add-on therapy to conventional therapy, not providing direct comparison of effectiveness of these innovative therapies themselves with conventional treatment [12].

Therefore, the aim of our study was to evaluate the effectiveness of additional therapy using the Armeo Spring robotic arm device compared to additional conventional physiotherapy for upper limb function in the early phase of post-stroke rehabilitation.

The incidence of stroke rapidly increases with age, doubling for each decade after the age of $55[13,14]$. Crude mortality and crude incidence of stroke are both positively correlated with the proportion of the population aged over 65 years [15].

Therapies with visual biofeedback use modern technologies that older patients are often not familiar or comfortable with. For this reason, we decided to compare the effectiveness of virtual reality therapy in stroke patients aged over 65 to that of younger patients.

\section{Materials and methods}

The study was carried out in the Stroke Rehabilitation Unit of the General University Hospital in Prague between January 2015 and June 2019. Patients were admitted from the
Department of Neurology early after acute stroke (days after stroke to baseline: mean $14.79 \pm 6.88 \mathrm{SD}$ ). The study was approved by the Ethics Committee of the General University Hospital and the patients signed informed consent before their entry into the study. Consecutively admitted patients to the rehabilitation department, fulfilling the inclusion criteria, were randomly assigned to either the virtual reality intervention group (IG) or to the control group (CG) with conventional physiotherapy, both in supplement to the daily rehabilitation programme. This programme consists of at least 3-4 hours of activity which includes one hour of physiotherapy twice a day, occupational therapy, therapies using passive or motor splints and motor operated/motor assisted/ active movement training for lower extremities. According to an individual patient's needs there is also group therapy for functional hand training and individual or group therapy for speech and cognitive impairment. Enrollment of the patients was complete when 25 patients had successfully finished the study in each group [IG $(n=25), C G(n=25)]$. Patients who didn't complete the full three-week programme for health reasons (unconnected to the rehabilitation programme) were excluded from the study.

Inclusion criteria were: first acute stroke with onset less than 30 days before the start of the therapy, ability to cooperate (as rated by the treating physician) and a post-stroke upper limb function deficit (FMA-UE 6-60 points). Patients unable to cooperate due to a severe hemispatial neglect syndrome (HSNS), usually connected with a low FMA-UE score, were excluded. Patients with mild HSNS (according to Catherine Bergego Scale (CBS) [16]) who could cooperate and use an Armeo device participated in the study.

Exclusion criteria were a severe cognitive impairment or severe sensoric aphasia, severe vision impairment diagnosed by an ophthalmologist, and the presence of any other neurological condition. MoCA scores were not used as an exclusion criterion. We used a neuropsychological assessment and a speech therapist assessment to assess the cognitive impairment and/or sensoric aphasia, as standardised cognitive tests didn't reflect the ability to understand and perform the therapy.

The primary outcome was measured by the Fugl Mayer Assessment Upper Extremity Scale (FMA-UE) [17-19]. The Functional Independence Measure (FIM) [20, 21] was chosen to assess secondary outcomes in the form of daily living activities. The Montreal Cognitive Assessment (MoCA) [22, 23] was performed to follow changes in cognitive functions. All assessments were performed at the beginning and end of a three-week therapy with 12 therapeutic sessions, when four conventional therapies were replaced by VRT in the IG group every week.

A total of 50 adults, mean age $67.34 \pm 12.21$ years, completed the study, of whom 29 were aged over 65 . Forty-four patients suffered from ischaemic stroke and six from haemorrhagic stroke (Tab. 1). Patients were initially treated by IVT/EVT or with no intervention (according to the time of stroke onset 
Table 1. Baseline characteristics of participants

\begin{tabular}{|c|c|c|c|c|c|c|c|c|c|c|}
\hline Baseline & $\mathbf{N}$ & $\begin{array}{c}\text { i-CVA / } \\
\text { h-CVA, } \\
n\end{array}$ & $\begin{array}{c}\text { Age, } \\
\text { years, } \\
\text { mean } \pm \text { SD }\end{array}$ & $\begin{array}{c}\text { Age } \\
\geq 65 \\
n\end{array}$ & $\begin{array}{c}\text { Gender } \\
\text { m/f, n }\end{array}$ & $\begin{array}{l}\text { DTB, days, } \\
\text { mean } \pm \text { SD }\end{array}$ & $\begin{array}{l}\text { Paresis of do- } \\
\text { minant /right/ } \\
\text { left hand, n }\end{array}$ & HSNS & $\begin{array}{c}\text { FMA-UE } \\
\text { mean } \pm \text { SD }\end{array}$ & $\begin{array}{c}\text { FIM } \\
\text { mean } \pm \text { SD }\end{array}$ \\
\hline Armeo IG & 25 & $20 / 5$ & $66.56 \pm 12.26$ & 14 & $14 / 11$ & $14.88 \pm 6.45$ & $16 / 18 / 7$ & 1 & $39.0 \pm 14.54$ & $89.04 \pm 14.35$ \\
\hline Armeo CG & 25 & $24 / 1$ & $68.12 \pm 11.97$ & 15 & $15 / 10$ & $16.4 \pm 7.25$ & $13 / 14 / 11$ & 4 & $45.2 \pm 15.52$ & $82.8 \pm 19.92$ \\
\hline
\end{tabular}

i-CVA/h-CVA — ischaemic/haemorrhagic cerebrovascular accident; DTB — days after stroke to baseline; HSNS — hemispatial neglect syndrome; FMA-UE — Fugl Mayer Assessment Upper Extremity Scale; FIM - Functional Independence Measure

Table 2. Assessment results

\begin{tabular}{|c|c|c|c|c|c|c|c|}
\hline \multirow[t]{2}{*}{ Armeo } & \multicolumn{2}{|c|}{ IG } & \multirow{2}{*}{$\begin{array}{c}n=\mathbf{2 5} \\
\mathbf{\Delta} \mathbf{\Delta} \%\end{array}$} & \multicolumn{2}{|c|}{ CG } & \multicolumn{2}{|l|}{$\mathrm{n}=\mathbf{2 5}$} \\
\hline & $\begin{array}{c}\text { To, mean } \\
\quad \pm \text { SD }\end{array}$ & $\begin{array}{c}\text { T1, mean } \\
\pm S D\end{array}$ & & $\begin{array}{c}\text { To, mean } \\
\quad \pm \text { SD }\end{array}$ & $\mathrm{T} 1$, mean $\pm \mathrm{SD}$ & $\Delta \Delta \%$ & p value \\
\hline $\operatorname{MoCA}(0-30)$ & $21.8 \pm 4.88$ & $25.6 \pm 3.54$ & $\begin{array}{c}3.8 \\
17.43\end{array}$ & $20.3 \pm 6.14$ & $22.9 \pm 5.53$ & $\begin{array}{c}2.6 \\
12.8\end{array}$ & 0.302 \\
\hline FIM (0-126) & $89.0 \pm 14.35$ & $110.8 \pm 8.17$ & $\begin{array}{c}21.8 \\
24.49\end{array}$ & $82.8 \pm 19.92$ & $104.9 \pm 15.49$ & $\begin{array}{c}22.1 \\
26.69\end{array}$ & 0.808 \\
\hline FMA-UE (0-66) & $39.0 \pm 14.54$ & $54.5 \pm 10.06$ & $\begin{array}{c}15.5^{*} \\
39.74^{*}\end{array}$ & $45.2 \pm 15.52$ & $54.2 \pm 13.93$ & $\begin{array}{c}9 \\
19.91\end{array}$ & $0.0209 *$ \\
\hline LOS, days, mean & & $35.76 \pm 10.79$ & & & $33.08 \pm 8.53$ & & 0.741 \\
\hline
\end{tabular}

IG - intervention group; CG — control group; MoCA — Montreal Cognitive Assessment; FIM — Functional Independence Measure; FMA-UE — Fugl Mayer Assessment Upper Extremity Scale; LOS — length of stay; $\mathrm{T} 0=$ baseline; $\mathrm{T} 1=$ end of treatment intervention; $\Delta=$ change between $\mathrm{T} 0$ and $\mathrm{T} 1 ; \Delta \%=$ percentage of change between $\mathrm{T} 0$ and $\mathrm{T} 1$ compared to baseline level $\mathrm{TO} ;{ }^{*}$ level of significance $95 \%$

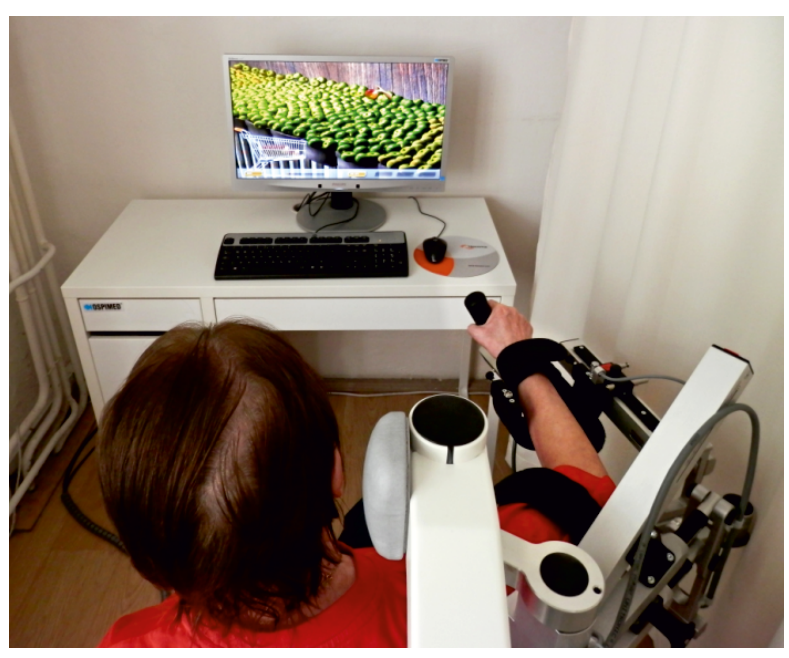

Figure 1. Armeo spring device

and other indications). Two participants (one each in IG and CG) dropped out of the study during the intervention period due to health problems not related to the exercise programme; they were replaced by further enrolled patients. Altogether, 29 men and 21 women completed the programme. There was no significant difference between the Intervention Group and the Control Group on the baseline of the study in terms of FMA-UE, FIM, age or days after stroke. There were four patients with mild HSNS in the control group compared to one patient in the intervention group. We used the Armeo Spring device [24, 25] (Fig. 1). This upper extremity exoskeleton gives weight support for the arm and hand, which allows the use of remaining motor functions. Movement is self-initiated. Reach and grasp are trained based on the patient's simultaneous movement of arm and hand. The therapist or patient can choose specific treatment goals using visual biofeedback on the screen in the form of games, completing different functional tasks [26].

Data for all age groups was analysed using MS Excel and $\mathrm{R}$ statistical software. Following the calculation of descriptive statistics, the hypothesis that the results in the IG and the results in the CG were selected from populations having the same distribution were tested by non-parametric Mann-Whitney $U$ test. To compare results for patients under 65 and over 65 , Fisher's exact test was used (due to small sample sizes).

\section{Results}

There was no difference in the magnitude of improvement in MoCA and FIM between the IG and CG groups. There was a significantly bigger improvement in FMA-UE for IG than for CG (p-value = 0.0209) (Tab. 2).

Comparing results for patients $<65$ and $\geq 65$ years old (Fisher's exact test), there was no significant difference between younger and older patients (Tab. 3) in terms of the distributions in MoCA and FMA-UE both in the IG and the CG (p-value between 0.14 and 0.68). As concerns FIM values, the hypothesis of equal distribution cannot be rejected for IG (p-value $=0.377$ ), while it is rejected for CG at a 95\% level of significance ( $\mathrm{p}$-value $=0.040$ ), which was caused by poor results of patients below 65 years (all eight patients reached FIM values below or equal to 30 ). 
Table 3. Results for patients over 65 compared to younger

\begin{tabular}{|c|c|c|c|c|}
\hline & $\begin{array}{c}\text { Armeo } \\
n=14\end{array}$ & $n=11$ & $n=17$ & $\mathbf{n}=\mathbf{8}$ \\
\hline Test (range) & $\begin{array}{c}\text { IG } \geq 65 \\
\Delta \text { mean } \\
\Delta \%\end{array}$ & $\begin{array}{c}\text { IG }<65 \\
\Delta \text { mean } \\
\Delta \%\end{array}$ & $\begin{array}{c}C G \geq 65 \\
\Delta \text { mean } \\
\Delta \%\end{array}$ & $\begin{array}{c}C G<65 \\
\Delta \text { mean } \\
\Delta \%\end{array}$ \\
\hline $\operatorname{MoCA}(0-30)$ & $\begin{array}{c}4.43 \\
20.88\end{array}$ & $\begin{array}{c}2.8 \\
12.33\end{array}$ & $\begin{array}{c}3.13 \\
16.20\end{array}$ & $\begin{array}{l}1.75 \\
7.90\end{array}$ \\
\hline FIM (0-126) & $\begin{array}{l}20.86 \\
23.10\end{array}$ & $\begin{array}{l}23.00 \\
26.24\end{array}$ & $\begin{array}{l}23.70 \\
29.54\end{array}$ & $\begin{array}{l}18.87 \\
21.38\end{array}$ \\
\hline FMA-UE (0-66) & $\begin{array}{c}13.5 \\
32.25\end{array}$ & $\begin{array}{l}17.81 \\
50.36\end{array}$ & $\begin{array}{c}9.11 \\
20.93\end{array}$ & $\begin{array}{c}8.62 \\
17.69\end{array}$ \\
\hline
\end{tabular}

IG - intervention group; CG - control group; MoCA - Montreal Cognitive Assessment; FIM — Functional Independence Measure; FMA-UE — Fugl Mayer Assessment Upper Extremity Scale; T0 = baseline; T1 $=$ end of treatment intervention; $\Delta=$ change between $\mathrm{T} 0$ and $\mathrm{T} 1 ; \Delta \%=$ percentage of change between $\mathrm{T} 0$ and $\mathrm{T} 1$ compared to baseline level $\mathrm{TO}$

\section{Discussion}

The aim of this randomised study was to evaluate the effectiveness of the VR therapy Armeo Spring upper limb exoskeleton in early post-stroke rehabilitation, as well as to investigate whether there were differences between younger (below 65 years) and older (over 65) patients. We analysed the influence of rehabilitation on reduction of final disability level. We did not take into consideration the initial impairment caused by stroke (NIHSS) nor the type of primary intervention (IVT/ EVT/no intervention). In the study by Angerova et al. [27], the cost-effectiveness of early inpatient rehabilitation after stroke is positively associated with the degree of initial motor disability.

The main finding of our study is that additional Armeo therapy had significantly better results than additional conventional therapy, particularly for upper extremity motor performance as assessed by the FMA-UE. The Armeo study secondary outcomes (MoCA and FIM) did not show any difference between the IG and CG.

There was a higher number of patients with mild HSNS in the control group, which could slightly influence the results $[28,29]$, but the numbers of HSNS were too small in both groups to form statistical evidence ( $\mathrm{p}$-value $=0.349$ for Fisher's exact test).

In the literature, most studies of robot-assisted upper limb rehabilitation (RT-UL) when added to conventional therapy have proved an effect on upper limb motor performance. For example, in the systematic review and meta-analysis of RT-UL in stroke rehabilitation by Norouzi-Gheidari [11], additional RT-UL is more beneficial, but when the duration time or intensity of the conventional therapy is matched to that with RT-UL, no difference was found between therapies in terms of motor recovery, ADL or motor control. The same results were found from the Cochrane Database of Systematic Reviews by Laver et al. [26]. Greater reductions in motor impairment and improvements in functional abilities were found in acute post-stroke patients who received RT-UL in addition to conventional therapy in the study of Masiero et al. [12]. In RCTs of Taveggia et al. [30], Colomer et al. [31] and Bartoldo et al. [32], the RT-UL time-matched to conventional therapy was found to be similarly effective (but not more effective) as conventional therapies. Also, Rodgers et al. [33] found in a multicentre randomised controlled trial with matched therapy time duration that results in RT-UL did not differ from control groups.

Only Veerbeek et al. [10] in their meta-analysis of relevant RCTs for RT-UL compared to non-robotic treatment found significant, but small, improvements in motor control and muscle strength of the paretic arm and a negative effect on muscle tone, while no effects were found for basic ADL.

These results correspond with those of our study, where we could see an impact of RT-UL on upper limb motor performance, but not transferred to ADL. The influence of spasticity which contributes much to upper extremity motor performance in the post-acute and chronic phases after stroke was not investigated in our study.

Our patients finished the study less than two months after stroke and only a small proportion of them developed mild spasticity MAS $\leq 2$ during this time that did not interfere with the function. This corresponds with our 2017 study [34]. None of the patients needed treatment of botulinum toxin on the upper hand in the time of the study. There is still not enough evidence on spasticity management at such an early stage, with most studies starting to treat spasticity three months after stroke onset. In the study by Schinwelski et al. [35] focused on 121 stroke patients with hemiparesis, $45 \%$ of patients developed spasticity (MAS $\geq 1$ ) in the three months after stroke. According to Wissel et al. [36], the treatment of focal or regional spasticity with intramuscular injections of botulinum toxin should start early in the 'golden time' when spasticity occurs and before soft tissues begin to shrink. Gracies $[37,38,39]$ recommends (besides botulinum toxin) to apply early prolonged stretching on hyperactive muscles and active repetitive movements on paretic ones. In our department, we use those principles immediately when spasticity occurs.

However, results in RCTs differ. It's important to continue studies that seek to identify appropriate stroke patients with a potential for recovery at the early post-stroke baseline. 
Calabro et al. [40] offered an evaluation of the cortical excitability and plasticity potential of the bilateral primary motor areas using transcranial magnetic stimulation to predict Armeo potential to improve upper limb motor function recovery.

To the best of our knowledge, there is a high number of studies on the effectiveness of VR in stroke patients in subacute and chronic states. In our study, we followed patients in the very early phase, recruited not later than one month from stroke onset. Such an early start of the therapies with VR or RT-UL is exceptional. In the meta-analysis of Veerbeek [10], the impact of RT-UL, if started in the first post-stroke weeks, remains unclear. Laver et al. [26] compared studies with patients recruited within six months after stroke to those recruited after six months or more. They found significantly better outcomes in IG (with VR) in the chronic phase, but not in the subacute one. According to the authors, the reason could be higher spontaneous recovery in both groups in subacute patients. Our results show moderate evidence of the effectiveness of VR in acute post-stroke patients for upper limb motor performance. We also assessed the effectiveness of therapy with VR in patients over 65 . There was no significant difference found between younger and older patients in all performed tests in IG or CG. Older adults using the Armeo device improved significantly more in FMA-UE compared to the CG, in the same way as younger participants.

There have been some papers investigating the use of virtual reality in the elderly, however we know of few studies comparing younger participants to the elderly. VR includes applications to promote physical activities, interactive video games, balance platforms and/or assist robots. A systematic review of active video games on rehabilitative outcomes among older patients by Zeng et al. [41] reported positive effects on balance, physical functioning and level of motivation. However, the existing evidence is insufficient to support the advantages of VR over the standard therapy. It is also unclear whether VR applications constitute a viable rehabilitative tool to improve cognitive outcomes.

\section{Clinical implications/future directions}

Therapies using virtual reality with visual biofeedback have similarly good results as those of conventional therapy in post-stroke patients when starting not later than 30 days after stroke onset. The Armeo Spring device has a good influence on upper extremity motor performance. Patients over 65 years do not have worse outcomes. Further studies with novel rehabilitation therapy techniques in larger patient samples are needed to confirm our preliminary data, as well as the timing and optimal length of treatment.

Disclosure: This project was supported by Specific Academic Research Projects Competition of Charles University in Prague No. 260500.

Conflicts of interest: None.
Abbreviations:

VRT - virtual reality therapies; IG - intervention group; CG - control group; MoCA - Montreal Cognitive Assessment; FIM - Functional Independence Measure; FMA-UE - Fugl Mayer Assessment Upper Extremity Scale; m-RIM - Modified Rivermead Mobility Index; RT - robot-assisted therapy; RT-UL - robot-assisted therapy for upper limb; VR — virtual reality; HSNS - hemispatial neglect syndrome; CBS - Catherine Bergego Scale; RCTs - randomised controlled trials; MAS - Modified Ashworth Scale

\section{References}

1. Lin MP. Time matters greatly in acute stroke care. Neurol Neurochir Pol. 2020; 54(2): 104-105, doi: 10.5603/PJNNS.2020.0037, indexed in Pubmed: 32352152.

2. Nowak K, Derbisz J, Jagiełła J, et al. Time from stroke onset to groin puncture affects rate of recanalisation after mechanical thrombectomy: a real-life single centre experience. Neurol Neurochir Pol. 2020; 54(2): 156-160, doi: 10.5603/PJNNS.a2020.0024, indexed in Pubmed: 32242914.

3. Winters C, van Wegen EEH, Daffertshofer A, et al. Generalizability of the Proportional Recovery Model for the Upper Extremity After an Ischemic Stroke. Neurorehabil Neural Repair. 2015; 29(7): 614-622, doi: 10.1177/1545968314562115, indexed in Pubmed: 25505223.

4. Zarahn E, Alon L, Ryan SL, et al. Prediction of motor recovery using initial impairment and fMRI $48 \mathrm{~h}$ poststroke. Cereb Cortex. 2011; 21(12): 2712-2721, doi: 10.1093/cercor/bhr047, indexed in Pubmed: 21527788.

5. Biernaskie J, Chernenko G, Corbett D. Efficacy of rehabilitative experience declines with time after focal ischemic brain injury. J Neurosci. 2004; 24(5): 1245-1254, doi: 10.1523/JNEUROSCI.3834-03.2004, indexed in Pubmed: 14762143.

6. Kwakkel G, van Peppen R, Wagenaar RC, et al. Effects of augmented exercise therapy time after stroke: a meta-analysis. Stroke. 2004; 35(11): 2529-2539, doi: 10.1161/01.STR.0000143153.76460.7d, indexed in Pubmed: 15472114.

7. Murphy TH, Corbett D. Plasticity during stroke recovery: from synapse to behaviour. Nat Rev Neurosci. 2009; 10(12): 861-872, doi: 10.1038/nrn2735, indexed in Pubmed: 19888284.

8. Kitago T, Krakauer JW. Motor learning principles for neurorehabilitation. Handb Clin Neurol. 2013; 110: 93-103, doi: 10.1016/B978-0444-52901-5.00008-3, indexed in Pubmed: 23312633.

9. Bertani R, Melegari C, De Cola MC, et al. Effects of robot-assisted upper limb rehabilitation in stroke patients: a systematic review with meta-analysis. Neurol Sci. 2017; 38(9): 1561-1569, doi: 10.1007/ s10072-017-2995-5, indexed in Pubmed: 28540536.

10. Veerbeek JM, Langbroek-Amersfoort AC, van Wegen EEH, et al. Effects of Robot-Assisted Therapy for the Upper Limb After Stroke. Neurorehabil Neural Repair. 2017; 31(2): 107-121, doi: 10.1177/1545968316666957, indexed in Pubmed: 27597165.

11. Norouzi-Gheidari N, Archambault PS, Fung J. Effects of robot-assisted therapy on stroke rehabilitation in upper limbs: systematic review and meta-analysis of the literature. J Rehabil Res Dev. 2012; 49(4): 479-496, doi: 10.1682/jrrd.2010.10.0210, indexed in Pubmed: 22773253.

12. Masiero S, Celia A, Rosati G, et al. Robotic-assisted rehabilitation of the upper limb after acute stroke. Arch Phys Med Rehabil. 2007; 
88(2): 142-149, doi: 10.1016/j.apmr.2006.10.032, indexed in Pubmed: 17270510.

13. Chong J, Sacco R. Risk factors for stroke, assessing risk, and the mass and high-risk approaches for stroke prevention. Continuum: Lifelong Learning in Neurology. 2005; 11: 18-34, doi: 10.1212/01. con.0000293708.61582.f4.

14. Ovbiagele B, Nguyen-Huynh MN. Stroke epidemiology: advancing our understanding of disease mechanism and therapy. Neurotherapeutics. 2011; 8(3): 319-329, doi: 10.1007/s13311-011-0053-1, indexed in Pubmed: 21691873.

15. Thrift AG, Thayabaranathan T, Howard G, et al. Global stroke statistics. Int J Stroke. 2017; 12(1): 13-32, doi: 10.1177/1747493016676285, indexed in Pubmed: 27794138.

16. Chen $\mathrm{P}$, Hreha $\mathrm{K}$, Fortis $\mathrm{P}$, et al. Functional assessment of spatial neglect: a review of the Catherine Bergego scale and an introduction of the Kessler foundation neglect assessment process. Top Stroke Rehabil. 2012; 19(5): 423-435, doi: 10.1310/tsr1905-423, indexed in Pubmed: 22982830.

17. Fugl-Meyer AR, Jääskö L, Leyman I, et al. The post-stroke hemiplegic patient. 1. a method for evaluation of physical performance. Scand J Rehabil Med. 1975; 7(1): 13-31, indexed in Pubmed: 1135616.

18. Gladstone DJ, Danells CJ, Black SE. The fugl-meyer assessment of motor recovery after stroke: a critical review of its measurement properties. Neurorehabil Neural Repair. 2002; 16(3): 232-240, doi: 10.1177/154596802401105171, indexed in Pubmed: 12234086.

19. Thompson-Butel AG, Lin G, Shiner CT, et al. Comparison of three tools to measure improvements in upper-limb function with poststroke therapy. Neurorehabil Neural Repair. 2015; 29(4): 341-348, doi: 10.1177/1545968314547766, indexed in Pubmed: 25209302.

20. Ottenbacher KJ, Hsu Y, Granger CV, et al. The reliability of the functional independence measure: a quantitative review. Arch Phys Med Rehabil. 1996; 77(12): 1226-1232, doi: 10.1016/s00039993(96)90184-7, indexed in Pubmed: 8976303.

21. Choo SX, Stratford P, Richardson J, et al. Comparison of the sensitivity to change of the Functional Independence Measure with the Assessment of Motor and Process Skills within different rehabilitation populations. Disabil Rehabil. 2018; 40(26): 3177-3184, doi: 10.1080/09638288.2017.1375033, indexed in Pubmed: 28891345.

22. MoCA test. https://www.mocatest (February 25, 2020).

23. Dong Y, Lee WY, Basri NA, et al. The Montreal Cognitive Assessment is superior to the Mini-Mental State Examination in detecting patients at higher risk of dementia. Int Psychogeriatr. 2012; 24(11): 1749-1755, doi: 10.1017/S1041610212001068, indexed in Pubmed: 22687278.

24. Armeo Spring Hocoma. https://www.hocoma.com/solutions/armeo-spring/ (February 25, 2020).

25. Gijbels D, Lamers I, Kerkhofs L, et al. The Armeo Spring as training tool to improve upper limb functionality in multiple sclerosis: a pilot study. J Neuroeng Rehabil. 2011; 8: 5, doi: 10.1186/1743-0003-8-5, indexed in Pubmed: 21261965.

26. Laver KE, Lange B, George S, et al. Virtual reality for stroke rehabilitation. Cochrane Database Syst Rev. 2017; 11: CD008349, doi: 10.1002/14651858.CD008349.pub4, indexed in Pubmed: 29156493.

27. Angerova Y, Marsalek P, Chmelova I, et al. , Cost and cost-effectiveness of early inpatient rehabilitation after stroke varies with initial disability, International Journal of Rehabilitation Research: September 24. 2020; 43(4): 376-382.

28. Parton A, Malhotra P, Husain M. Parton A, Malhotra P, Husain M, Hemispatial neglect, Journal of Neurology, Neurosurgery \& Psychiatry. 2004; 75: 13-21.

29. Di Monaco M, Schintu S, Dotta M, et al. Severity of unilateral spatial neglect is an independent predictor of functional outcome after acute inpatient rehabilitation in individuals with right hemispheric stroke. Arch Phys Med Rehabil. 2011; 92(8): 1250-1256, doi: 10.1016/j. apmr.2011.03.018, indexed in Pubmed: 21807144.

30. Taveggia G, Borboni A, Salvi L. at al. Efficacy of robot-assisted rehabilitation for the functional recovery of the upper limb in post-stroke patients: a randomized controlled study. Eur J Phys Rehabil Med. 2016; 52(6): 767-773.

31. Colomer C, Baldoví A, Torromé S, et al. Efficacy of Armeo® Spring during the chronic phase of stroke. Study in mild to moderate cases of hemiparesis. Neurologia. 2013; 28(5): 261-267, doi: 10.1016/j. nrl.2012.04.017, indexed in Pubmed: 22727271.

32. Bartolo M, De Nunzio AM, Sebastiano F, et al. Arm weight support training improves functional motor outcome and movement smoothness after stroke. Funct Neurol. 2014; 29(1): 15-21, indexed in Pubmed: 25014045

33. Rodgers $\mathrm{H}$, Bosomworth $\mathrm{H}$, Krebs $\mathrm{H}$, et al. Robot assisted training for the upper limb after stroke (RATULS): a multicentre randomised controlled trial. The Lancet. 2019; 394(10192): 51-62, doi: 10.1016/ s0140-6736(19)31055-4.

34. Gueye T, Dedkova M. Outcome of the Armeo Therapy with the Patients Developing Spastic Paresis of the Upper Limb, IV. Kladruby Symposium on Interdisciplinary Neurorehabilitation, book of abstracts, 2017, ISBN: 978-80-270-2951-8.

35. Schinwelski MJ, Sitek EJ, Wąż P, et al. Prevalence and predictors of post-stroke spasticity and its impact on daily living and quality of life. Neurol Neurochir Pol. 2019; 53(6): 449-457, doi: 10.5603/PJNNS. a2019.0067, indexed in Pubmed: 31845749.

36. Wissel J, Ward AB, Erztgaard P, et al. European consensus table on the use of botulinum toxin type A in adult spasticity. J Rehabil Med. 2009; 41(1): 13-25, doi: 10.2340/16501977-0303, indexed in Pubmed: 19197564.

37. Gracies JM. Pathophysiology of spastic paresis. I: Paresis and soft tissue changes. Muscle Nerve. 2005; 31(5): 535-551, doi: 10.1002/ mus.20284, indexed in Pubmed: 15714510.

38. Gracies JM, Bayle N, Vinti M, et al. Five-step clinical assessment in spastic paresis. Eur J Phys Rehabil Med. 2010; 46(3): 411-421, indexed in Pubmed: 20927007.

39. Gracies JM. Pathophysiology of Impairment in Patients with Spasticity and Use of Stretch as a Treatment of Spastic Hypertonia. Physical Medicine and Rehabilitation Clinics of North America. 2001; 12(4): 747-768, doi: 10.1016/s1047-9651(18)30031-7.

40. Calabrò RS, Russo M, Naro A, et al. Who May Benefit From Armeo Power Treatment? A Neurophysiological Approach to Predict Neurorehabilitation Outcomes. PM R. 2016; 8(10): 971-978, doi: 10.1016/j. pmrj.2016.02.004, indexed in Pubmed: 26902866.

41. Zeng N, Pope Z, Lee J, et al. A systematic review of active video games on rehabilitative outcomes among older patients. Journal of Sport and Health Science. 2017; 6(1): 33-43, doi: 10.1016/j.jshs.2016.12.002. 